\title{
Flâner ou filer : une approche quantifiée de l'influence du contexte sur le type de pratique piétonne
}

LVMT, UMR-T 9403, École des Ponts, IFSTTAR, UPEM, UPE, Champs-sur-Marne, France 6-8 avenue Blaise Pascal 77455 Marne La Vallée cedex 2,

julie.chretien@enpc.fr 


\title{
Flâner ou filer : une approche quantifiée de l'influence du contexte sur le type de pratique piétonne
}

\section{RESUME}

La marche est successivement et parfois simultanément un moyen de transport et une activité de loisir. Il est utile de comprendre l'articulation de ces deux facettes dans la mesure où le sens que les acteurs urbains lui donnent peut influencer les politiques publiques qui y sont consacrées. Cet article vise une meilleure connaissance du poids respectif de la «marche-loisir» et de la «marche utilitaire » en milieu urbain et de l'influence du cadre spatial et temporel du déplacement sur la prévalence des deux logiques. À l'aide de l'Enquête Globale Transport 2010 nous montrons qu'à la différence de la marche utilitaire, la marche-loisir n'est pas influencée par la densité du cadre urbain mais augmente quand les contraintes d'emploi du temps diminue. Cependant, les quatre cinquièmes des déplacements à pied relèvent d'une pratique utilitaire, ce qui invite à repenser la place prépondérante occupée par la marche-loisir dans les discours et dans les politiques de promotion de la marche.

Mots-clés : marche à pied, loisir, mobilité durable, aménagement, déplacement

\section{Taking a stroll or a trip: a quantitative approach to the context's influence on the type of pedestrian mobility}

\begin{abstract}
Walking is successively and sometimes simultaneously a means of transport and a leisure activity. In so far as the way urban actors perceive pedestrian practices can have consequences on the public policies that are devoted to it, it is useful to gain insight on the articulation of these two aspects. This article aims at a better knowledge of the proportion of walking trips in urban environment that are done for leisure and of the influence of the spatial and temporal context of the trip on the prevalence of the two logics. Using the 2010 Transport Survey of the Parisian Region, we show that, unlike utilitarian pedestrian practices, leisure practices are not influenced by the density of the urban setting, but increase when time-use constraints decrease. However, fourfifths of pedestrian journeys are utilitarian, which calls for a rethinking of the importance given to recreational pedestrian practices in policies which promote walking.
\end{abstract}

Keywords: walking, leisure, sustainable mobility, urban planing, transportation 


\section{INTRODUCTION}

La marche est un objet particulier dans le domaine de la mobilité, étant successivement (et parfois simultanément) moyen de transport et activité à part entière. Or le sens que les décideurs et les usagers donnent à la pratique piétonne peut avoir des conséquences sur les politiques d'aménagement et de mobilité qui y sont consacrées. Cela suppose donc que ces acteurs aient une connaissance équilibrée de ces deux aspects de la marche et des facteurs qui peuvent favoriser l'un ou l'autre.

Actuellement, de quelle manière les deux facettes de la marche sont-elles perçues dans les politiques de transport et d'aménagement et dans les représentations sociales ? Dans des politiques de mobilité, la marche apparait principalement à travers l'étude des populations défavorisées qui n'ont pas d'alternative, l'objectif étant alors de fournir une voiture aux ménages qui n'en ont pas, plutôt qu'à augmenter le nombre de services accessibles à pied (Bacqué et Fol 2007). La marche y est ainsi perçue comme un pis-aller, auquel être cantonné limiterait les chances d'insertion. Quant aux politiques d'aménagement, leur prise en compte de la marche prend la forme d'une grande attention accordée au design des espaces (Hess et al. 1999 ; Bostock 2001 ; Genre-Grandpierre 2007 ; Lavadinho et Winkin 2012), soulignant ainsi en creux l'importance supposée de l'expérience vécue comme facteur déterminant dans sa pratique. Enfin, Monnet (2015) montre qu'il existe dans le champ de l'imaginaire social une « marcheloisir», noble et valorisée, opposée à une «marche-déplacement» souvent ignorée de la littérature. Dans les discours, domine ainsi l'image de la marche comme activité en soi : son rôle en tant que moyen de transport indispensable à la vie quotidienne de grands pans de la population est largement occulté et déconsidéré en dehors des espaces aisés ou touristiques.

Ces vingt dernières années, des travaux ont tenté d'extraire la marche en ville de sa relative invisibilité ou de son cantonnement aux populations sous-motorisées. Cependant, ceux-ci n'ont pas clarifié l'ambiguïté inhérente à cette pratique. Ils l'ont soit traitée en tant que moyen de transport en soulignant son caractère efficace dans de nombreuses situations, mais laissant de côté l'agrément qu'elle peut représenter (Papon et De Solère 2010; Lejoyeux et Laousse 2010; Chrétien 2015), soit ont souligné à quel point la pratique piétonne est appréciée et associée à des sentiments de plaisir, subsumant le caractère utilitaire des trajets piétons sous l'expérience de vie qu'ils représentent (Thomas 2004 ; Simon 2011).

La permanence de cette dichotomie, même au sein d'articles visant à mieux objectiver les pratiques, nous amène à faire l'hypothèse que les outils employés par les chercheurs orientent vers l'une ou l'autre pratique des pratiques piétonnes. Lors d'entretiens semi-directifs, la marche-loisir étant la seule valorisée, ce sont ses récits qui émergent, ceux autour de la marche-déplacement étant difficiles à saisir (Augoyard 1979). En retour, les outils utilisés pour analyser les flux de déplacements - et notamment parmi eux les Enquêtes Ménage Déplacement (EMD) - sont construits pour optimiser des transports fonctionnels et se basent sur une conception normée de la mobilité (Commenges 2013) distinguant les déplacements des activités : chaque déplacement y correspond au mouvement entre une origine et une destination, chaque destination étant décrite par un " motif », c'est-à-dire ce que la personne compte y faire. Ainsi ces enquêtes, dans leur construction même, n'admettent le déplacement que comme moyen, ce qui rend difficile l'analyse des cas où ceux-ci sont des fins en soi.

Par conséquent, si la marche-déplacement a été en partie revalorisée, cela s'est fait au prix d'une tension entre deux visions des pratiques piétonnes. Or on peut faire l'hypothèse que l'ambivalence des regards portés sur la marche n'est que le reflet d'une pratique elle-même ambivalente. Il apparaît dès lors nécessaire de développer une analyse interprétant conjointement ces deux facettes, ce qui permettrait d'une part d'estimer la prévalence de chaque logique et d'autre part de comprendre les facteurs faisant émerger l'une ou l'autre.

Les outils théoriques utilisés en socio-économie des transports permettent de penser la dualité de la marche. Si le modèle dominant dans l'analyse de la mobilité considère que le mouvement physique n'est qu'un moyen d'atteindre une destination distante dans l'espace et que ce temps est un coût à minimiser afin de maximiser l'utilité de l'activité, des auteurs ont entrepris d'en contester l'universalité en soulignant qu'une valeur positive peut être accordée au fait de se déplacer. Mokhtarian et Salomon (2001) montrent qu'il existe un ensemble de situations (randonnées, promenades, croisières...) où le déplacement représente un moment utile ou plaisant, et devient une activité à part entière. Il est possible de se placer dans des cas de figure où le temps de déplacement prend une valeur positive et où les individus ne cherchent pas à l'optimiser. Par conséquent, lorsqu'une logique d'évasion, de sociabilité, d'émotion (Flamm 2004) ou, de façon moins ambitieuse, une logique de minimisation de l'inconfort du déplacement est intégrée au déplacement, cela influence le choix du mode de transport et le temps consacré au trajet.

Cette approche théorique a en outre l'avantage de permettre d'utiliser des outils habituellement employés pour analyser la facette « mode de transport » de la marche pour identifier des logiques piétonnes de loisirs. En effet, en se plaçant dans hypothèse d'individus cherchant à maximiser leur utilité quotidienne, l'existence de trajets faits avec un mode qui n'est pas optimal temporellement suppose que d'autres facteurs sont intervenus pour contrebalancer ce « surcoût ». Soit que la personne ait refusé de payer le coût monétaire pour prendre un mode plus 
rapide, soit que des éléments relevant de l'expérience du trajet - confort, plaisir lié à l'environnement... - aient biaisé la perception du temps de trajet amenant à un rallongement de celui-ci, soit que la personne ait volontairement décidé de consacrer plus de temps à se déplacer pourvu que le moment soit plus agréable. Il est alors possible de « lire » dans les EMD les logiques de loisirs implicites de certains déplacements, notamment piétons, en identifiant les cas où les individus n'ont pas fait un choix de mode de transport optimisant le temps de déplacement.

Ainsi, en se plaçant dans ce cadre théorique, il est possible de voir la marche comme un mode de transport pour lequel la valeur du temps serait ambivalente, tantôt positive - fusse uniquement par rapport à d'autres modes de transport moins appréciés -, tantôt négative. La question se pose alors d'identifier les facteurs qui induisent ces variations.

Les enquêtes ad hoc menées pour analyser la valeur associée à l'activité de se déplacer révèlent que celleci n'est ni intrinsèque à un individu ni à un mode, mais qu'elle dépend des circonstances temporelles et spatiales du trajet. Ainsi, à travers une étude sur la région de San Francisco, Mokhtarian et Salomon (2001) montrent que $55 \%$ des enquêtés déclarent apprécier leur trajet lorsqu'ils se rendent sur un lieu de loisir, contre seulement $15 \%$ lorsqu'il s'agit d'accompagner quelqu'un. Anable et Gatersleben (2005) indiquent que lorsqu'ils se déplacent pour motifs professionnels, les individus accordent une plus grande importance aux éléments instrumentaux (le caractère pratique, la fiabilité, la flexibilité et le coût) dans le choix de leur mode de transport qu'aux éléments liés au plaisir, ce qui n'est pas le cas pour les trajets de loisir. Ces travaux rejoignent une vaste littérature sur la valeur du temps de déplacement, qui constate que le temps de transport « coûte moins cher $»^{1}$ lorsque l'on se déplace pour se rendre à des loisirs que pour des motifs professionnels (Crozet 2005). Il est ainsi possible de faire l'hypothèse que la marche soit pratiquée selon une logique de déplacement «pur » lorsque les individus doivent se rendre à des activités contraintes en termes d'horaires et d'obligation de présence, mais selon une logique de loisir lorsqu'ils disposent de plus de temps ou se rendent à des activités moins contraintes.

Si l'on peut faire l'hypothèse qu'augmenter les contraintes liées à l'emploi du temps amène à des pratiques plus purement «de déplacement», la question se pose de ce qui, à l'inverse, peut augmenter l'utilité tirée de la marche. Lorsque l'on interroge directement les individus au sujet des raisons les poussant à rallonger leurs trajets pas seulement à pied -, les raisons principales avancées ont trait à l'environnement du déplacement (Mokhtarian et Salomon 2001). Les nombreux travaux s'appuyant sur des préférences révélées pour identifier les façons dont l'aménagement peut favoriser les pratiques piétonnes insistent sur l'importance du sentiment de sécurité (Hess et al. 1999), sur la connectivité des rues (Genre-Grandpierre et Foltête 2003) et la qualité de l'environnement (Handy, Cao, et Mokhtarian 2005 ; Lavadinho et Winkin 2012). On assiste ainsi à combinaison de facteurs améliorant l'accessibilité offerte par la marche - et donc potentiellement ses usages « déplacement »-et de facteurs jouant sur l'agrément, pouvant inciter à une logique de « loisir » plus forte. Or Lo (2009) suggère que ces facteurs peuvent entrer en contradiction les uns avec les autres : un environnement dense peut rendre la marche plus efficace en tant que mode de transport, mais induire des phénomènes de foule diminuant son caractère plaisant. Par conséquent s'il a été montré que la densité et la mixité fonctionnelle augmentent les pratiques piétonnes, il n'est pas impossible que cela se fasse au détriment de logiques de loisir.

Ainsi si l'on part de l'hypothèse de travail que les individus cherchent à maximiser leur utilité au quotidien, on peut supposer que les circonstances temporelles et spatiales du trajet tendraient à renforcer soit une logique « utilitaire » (ou de déplacement « pur ») visant à minimiser le temps de transport (quitte, dans certains cas, à en payer le prix monétaire et en inconfort), soit une logique de « loisir» dans le cadre de laquelle les éléments liés à l'expérience positive de la marche domineraient. Nous chercherons dans cet article à révéler les situations les plus propices à chaque pratique piétonne et leur importance relative en fonction des circonstances.

\section{DONNEES ET METHODE}

\section{Données}

Nous nous appuierons sur l'Enquête Globale Transport de 2010 (EGT 2010), qui porte sur les déplacements des résidents d'Île-de-France. Les individus y sont invités à raconter leur mobilité au cours d'une journée, laquelle est ensuite traduite en une succession de déplacements. Le choix du terrain est lié à notre thèse. Il comporte en outre deux avantages pour l'étude des déplacements piétons. Premièrement, l'Île-de-France est une région dense et fortement urbanisée au sein de laquelle la marche est très pratiquée, ce qui fournit un échantillon analysable d'une ampleur conséquente. Secondement, lors de l'édition 2010, une attention particulière avait été portée aux déplacements piétons, les enquêteurs étant invités à relancer les enquêtés sur les trajets de faible portée. En outre, le carroyage employé est de $100 \mathrm{~m}$ par $100 \mathrm{~m}$. On est donc en mesure d'espérer que les déplacements piétons sont fidèlement représentés. Nous nous intéresserons uniquement aux personnes adultes, les mineurs ayant des

\footnotetext{
${ }^{1} \mathrm{Ou}$ dit autrement, « que l'on cherche moins à minimiser son temps de transport ».
} 
contraintes spécifiques sur leur accès à la mobilité. En raison des contraintes inhérentes à la base de données que nous exploitons, qui n'interroge que les personnes qui résident en Île-de-France, nous ne nous intéressons pas aux pratiques des touristes et autres non résidents de la région. Enfin, nous nous concentrerons ici sur les pratiques lors d'un jour de semaine (lundi à vendredi), le week-end étant moins bien représenté dans l'enquête.

Nous avons divisé l'Île-de-France en six zones comme suit. Tout d'abord, nous avons séparé Paris, une zone regroupant les communes appartenant à une unité urbaine ${ }^{2}$ que nous avons nommée «banlieue », et une zone regroupant les communes n'appartenant pas à une unité urbaine, que nous avons nommée "périurbain ». Comme $70 \%$ de notre population résidait dans la catégorie «banlieue », nous avons distingué entre les $20 \%$ des communes les plus denses (plus de 6000 habitants $/ \mathrm{km}^{2}$ ) et les autres. Enfin, à l'échelle du carroyage, nous avons distingué les lieux selon qu'ils se trouvent à plus ou moins de $800 \mathrm{~m}$ d'une gare (RER ou train) ou d'une station de métro ${ }^{3}$. Quand nous analyserons l'influence du lieu, nous distinguerons les déplacements en fonction de leur lieu d'origine.

Dans cet article, nous considérerons deux formes de marche-loisir, mutuellement exclusives. La première correspondra aux cas où le motif à destination est explicitement désigné comme étant la marche (les « promenades »). La seconde concernera les cas où la marche-loisir prend la forme d'un trajet qui mène quelque part, mais où la vitesse relative plus basse des trajets à pied par rapport aux autres modes suggère qu'une logique de plaisir est à l'œuvre (la marche en tant que «mode d'agrément»). Par contraste, nous définirons la «marche utilitaire » comme correspondant aux cas où la marche est le mode le plus rapide pour se rendre à la destination que l'on souhaite atteindre. Nous allons clarifier ces cas.

\section{Lorsque la marche est une activité à part entière}

Les promenades sont mises sous le même intitulé que le lèche-vitrines et les cours de conduite ${ }^{4} .8,7 \%$ de la population adulte d'Île-de-France a réalisé un déplacement pour un de ces motifs. Cependant, ce chiffre peut inclure des promenades en voiture ou en vélo ainsi que des cours de conduite. Afin de nous assurer autant que possible que nous ne saisissons que les cas de déplacements piétons, nous ne retenons que les cas où le mode emprunté pour ce déplacement est la marche et où la durée à destination est de quinze minutes ou moins. Nous perdons ici potentiellement les cas où les individus se rendent en voiture dans un centre-ville où ils feront du lèchevitrine à pied. On a donc là une limite basse de la prévalence de la pratique de la marche en tant qu'activité à part entière.

Par ailleurs, dans l'EGT 2010, les promenades, lèche-vitrines et cours de conduite sont codés comme suit : l'enquêteur indique la portée maximale de la promenade (ou de la séance de lèche-vitrines ou du cours de conduite), associe cette portée maximale à une destination à laquelle il est considéré que l'individu ne restera pas et répartit le temps en mouvement entre deux déplacements - un aller et un retour - créés de façon artificielle. Ainsi, une unique promenade est décomposée en deux déplacements. Par conséquent au lieu de fournir la part des déplacements dont la destination est une promenade, nous préciserons l'ensemble des déplacements ayant ce motif à l'origine ou à destination.

\section{Lorsque la marche sert à se rendre à une autre activité}

En dehors du cas où la marche est une activité en tant que telle, nous cherchons à identifier les situations où les individus avaient un motif de déplacement indépendant du trajet, mais où le plaisir de marcher a influencé le choix de mode de transport. En maintenant l'hypothèse d'individus cherchant à maximiser leur utilité quotidienne - hypothèse présentée en introduction -, il nous est possible de déduire la logique du trajet en identifiant les cas où la marche n'est pas le mode qui permet de minimiser le temps de transport.

Ce que l'on identifie de cette manière comme des pratiques de mode d'agrément pourraient relever d'une forme de captivité de la marche. Afin de nous assurer que ces cas sont secondaires, nous distinguerons au sein de notre population les individus qui ont déclaré qu'ils n'avaient pas de voiture à disposition le jour de l'enquête (et, pour les habitants de Paris et de la banlieue dense avec gare, qu'ils n'avaient pas non plus d'abonnement aux transports en commun), que nous appellerons piétons «captifs», du reste de la population, qualifiée de «motorisée ».

Dans ce contexte, l'enjeu est d'identifier sur quelles distances (que nous traduisons en temps de trajet piéton), la marche représente le mode plus optimal temporellement pour réaliser un trajet. Les deux tiers (67\%) des

\footnotetext{
${ }^{2}$ Dans plus de $90 \%$ des cas, il s'agit de l'unité urbaine de Paris.

${ }^{3}$ Cette distance équivaut environ à 10 minutes de marche et correspond au périmètre retenu comme étant sous l'influence de la gare, c'est à dire plus dense et diversifié que les alentours (voir les travaux de l'APUR sur les gares du Grand Paris Express et la note rapide de l'IAU n ${ }^{\circ} 762$ sur l'articulation entre gare et quartier de gare).

${ }^{4}$ Correspondant au motif à destination « 42. Promenade, lèche-vitrines [sans achat], leçons de conduite ».
} 
déplacements piétons durent 10 minutes ou moins, et $89 \%$ d'entre eux durent 20 minutes ou moins. Ceci suggère que les résultats seront très sensibles au choix du seuil temporel en deçà duquel la marche serait le mode optimal et que toute erreur de quelques minutes aurait un impact considérable sur les résultats. Par ailleurs l'incertitude du seuil se rajouterait à celle de la fiabilité des déclarations des individus, car $90 \%$ des durées déclarées pour la marche l'ont été à 5 minutes près. Enfin, comme ce seuil dépend de la performance des autres modes de transport et donc du moment de la journée et des lieux d'origine et de destination du déplacement, leur estimation supposerait donc une connaissance fine des conditions de trafic au lieu et à l'instant de chaque trajet. Par conséquent, nous cherchons plutôt une valeur nous permettant de fournir une limite basse aux pratiques de marche-loisir.

\section{Déterminer une limite basse aux pratiques de marche-loisir}

Dans Paris et en banlieue dense proche d'une gare, entre le temps d'attente en station et, le cas échéant (pour les modes ferrés), d'accès et d'exit, les transports en commun ne sont pas pertinents, dans le meilleur des cas, pour les déplacements de moins d'un kilomètre, soit, si l'on estime à $4 \mathrm{~km} / \mathrm{h}$ la vitesse de marche, ne sont pas pertinents en deçà de 15 minutes de trajet piéton. La voiture est moins performante encore que les transports en commun : si l'on estime à $15 \mathrm{~km} / \mathrm{h}$ la vitesse moyenne de déplacement automobile dans la capitale et à 13 minutes la somme du temps d'accès au véhicule et de recherche d'une place de parking (Lefauconnier et Gantelet 2005), en deçà d'un trajet de portée de 1,2 km (soit 18 minutes à pied) il n'est pas pertinent d'utiliser l'automobile.

Dans les zones moins denses (périurbain et banlieue à plus de $800 \mathrm{~m}$ d'une gare), estimer une distance sur laquelle la marche est le mode le plus rapide est une entreprise plus hasardeuse. Dans la majorité des cas, sur les petites distances, seule la voiture est compétitive car sa vitesse moyenne y est plus élevée. Cependant, bien plus que cette dernière, c'est le temps d'accès au véhicule qui détermine le périmètre à l'intérieur duquel la marche est optimale en temps. À titre d'exemple, en maintenant l'hypothèse d'une vitesse à $15 \mathrm{~km} / \mathrm{h}$, si le temps d'accès et de dépôt du véhicule chute à 2 minutes (ce qui représente une hypothèse crédible, voire haute, pour les ménages disposant d'un garage et se rendant en un lieu où les places sont nombreuses), la marche n'est optimale que sur $200 \mathrm{~m}$, c'est-à-dire sur des trajets de 3 minutes. Or ce temps d'accès dépend certes de la densité (très hétérogène parfois au sein d'une même commune), mais aussi de la politique de la commune en matière de stationnement et de l'heure du déplacement.

Retenons simplement que ce seuil en deçà duquel la marche est optimale sera maximal dans Paris. Par conséquent, nous retenons la valeur estimée pour cette zone - 15 minutes - comme seuil de référence fournissant une estimation basse de la prévalence de la marche-loisir : les trajets piétons dont la durée déclarée est inférieure ou égale à cette valeur seront considérés comme relevant d'une pratique utilitaire de la marche. Avec cette mesure, la marche-loisir sera estimée au plus proche dans Paris, mais certainement sous-estimée dans les zones moins denses, notamment dans les zones que nous qualifions de « périurbain », ce dont nous tiendrons compte dans notre analyse.

\section{RESULTATS}

\section{Prévalence des différentes pratiques piétonnes en Île-de-France}

Au sein de notre population, la marche représente $36 \%$ des déplacements réalisés et $49 \%$ des adultes résidant dans la région ont fait au moins un déplacement intégralement piéton le jour de l'enquête. La marche-loisir représente au moins $21 \%$ de ces déplacements, dont $9 \%$ de promenades et $12 \%$ où la marche est un mode d'agrément.

Les piétons « captifs » ont réalisé $29 \%$ des trajets piétons et représentent $20 \%$ de notre population, ils ont donc une pratique plus marquée de la marche que le reste des Franciliens adultes. Cependant, parmi les déplacements piétons (hors promenades) réalisés par les captifs, $84 \%$ relevaient d'une logique utilitaire, contre $87 \%$ chez les non-captifs. Si l'absence de véhicule motorisé incite donc à réaliser des trajets plus longs, cet impact est à nuancer : le comportement des personnes non motorisées est très proche de celui des personnes qui disposaient d'un véhicule.

\section{La marche-loisir dépend peu du lieu}

La prévalence de la marche dans son ensemble augmente fortement avec la densité de la zone de départ (Figure 1) : moins d'un déplacement sur cinq prenant son origine dans le périurbain se fait à pied, contre $30 \%$ de ceux partant de banlieue peu dense à moins de $800 \mathrm{~m}$ d'une gare et près de la moitié des déplacements ayant pour origine Paris. Cette hausse de la pratique de la marche est très majoritairement stimulée par la marche utilitaire, et ce en dépit d'une surestimation de cette dernière dans les zones moins denses.

La marche loisir en revanche n'augmente que faiblement avec la densité, et ce bien que nous la sousestimions dans les zones moins denses en prenant le seuil de 15 minutes. La pratique récréationnelle de la marche 
est ainsi stable et de faible ampleur : quel que soit le lieu, elle ne forme qu'entre 5 et $10 \%$ des déplacements tous modes confondus, ce qui représente une part relativement marginale des trajets quotidiens.

Figure 1 : Type de marche en fonction du lieu d'origine des déplacements

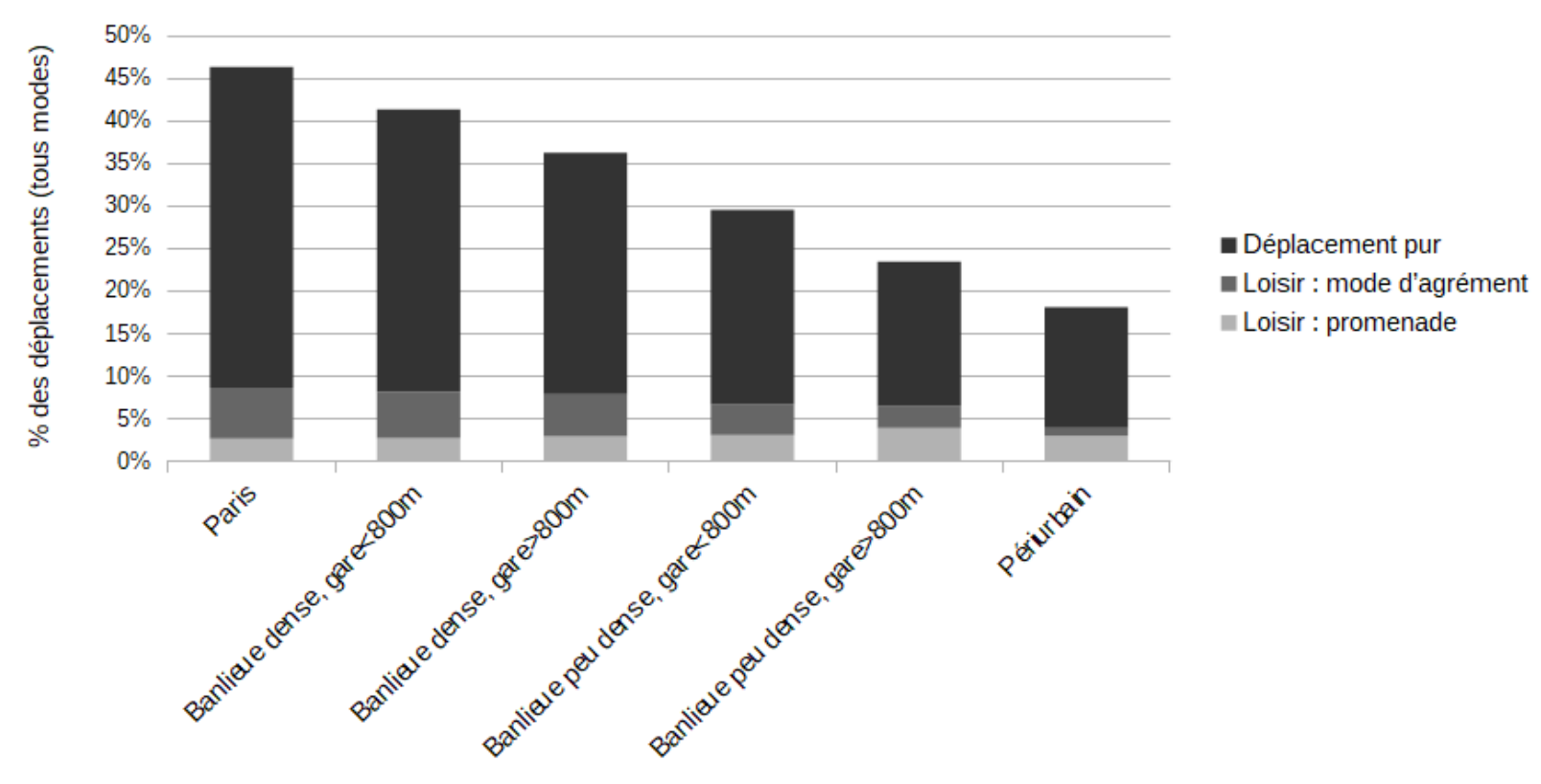

Zone d'origine du déplacement

Dans quelles proportions augmenter le seuil dans les zones peu denses y modifierait-il la proportion de marche-loisir? Si, en banlieue peu dense loin d'une gare et dans le périurbain, on prend 5 minutes pour seuil en deçà duquel la marche est optimale, la marche en tant que mode d'agrément représente respectivement $11 \%$ et $7 \%$ des déplacements, ce qui est une surreprésentation par rapport au taux observé à Paris (6\%), mais ne remet pas en cause la logique utilitaire dans ces territoires.

Par ailleurs, distinguer les piétons captifs des motorisés confirme les résultats agrégés observés dans la section précédente : les «motorisés » ont une pratique de la marche utilitaire non significativement supérieure à celle des captifs. À lieu de déplacement identique, les personnes qui ne possèdent pas de mode motorisé ne compensent pas en pratiquant plus de longs déplacements piétons. Ce résultat, contre-intuitif au moins pour ce qui concerne les zones moins denses, est cohérent avec les travaux de Benjamin Motte-Baumvol (2007, 2010), qui montrent que les ménages non motorisés sont presque absents des territoires les plus dépendants de l'automobile et se relocalisent dans des zones plus denses en services et commerces lorsqu'elles en ont l'occasion.

Ainsi, lorsque la densité baisse, la marche devient moins performante par rapport à d'autres modes de transports et l'on assiste à une diminution rapide de sa pratique. Là où l'on pourrait imaginer qu'une forte utilité positive maintiendrait une pratique piétonne même dans les zones où la marche est peu adaptée, poussant les individus à rallonger certains de leurs trajets pour pouvoir les réaliser à pied, c'est le phénomène inverse qui se produit : la durée médiane des trajets piétons est identique à travers l'Île-de-France, et chute même dans le périurbain. Par conséquent, la prévalence de la marche-loisir n'augmente pas lorsque de la densité du territoire diminue : le gain que peuvent apporter des cadres de déplacements plus apaisés ne compense pas le surcoût en temps qu'impliquent des déplacements piétons dans ces zones.

\section{Des pratiques de loisir qui augmentent quand la valeur du temps diminue}

De même que la marche en tant que mode d'agrément représentait une part marginale des déplacements, et ce indépendamment du lieu, elle demeure marginale quel que soit le motif du déplacement (Figure 2) : sa pratique représente au mieux $7 \%$ des déplacements tous modes confondus (pour les trajets domicile-affaires personnelles).

En revanche à la différence du lieu, le motif du déplacement - et par extension les circonstances temporelles de celui-ci - joue sur la prévalence de la marche en tant que mode d'agrément (Figure 2). Les quatre motifs combinés (hors motif «Domicile<->Autres ») pour lesquels la part modale de la marche en tant que mode d'agrément est le 
plus faible sont, par ordre croissant, les trajets domicile-travail, les trajets secondaires liés au travail, les trajets domicile-études et les accompagnements ${ }^{5}$. On remarque ainsi que les trajets émanant d'activités représentant des contraintes pour l'emploi du temps de l'individu sont ceux pour lesquels les pratiques de loisir sont les plus faibles.

La pratique de la marche utilitaire en revanche ne dépend pas des circonstances temporelles : on trouve parmi les motifs contraints aussi bien des trajets pour lesquels elle est rare que des trajets pour lesquels elle est fréquente. Par exemple, les trajets domicile-travail ou études sont très rarement concernés par la marche utilitaire ${ }^{6}$, alors que les déplacements secondaires liés au travail et les trajets domicile-accompagnement - contraints eux aussi - sont très fréquemment réalisés à pied dans une logique de déplacement pur.

Figure 2 : Type de pratique piétonne en fonction des motifs de déplacement (hors promenade)

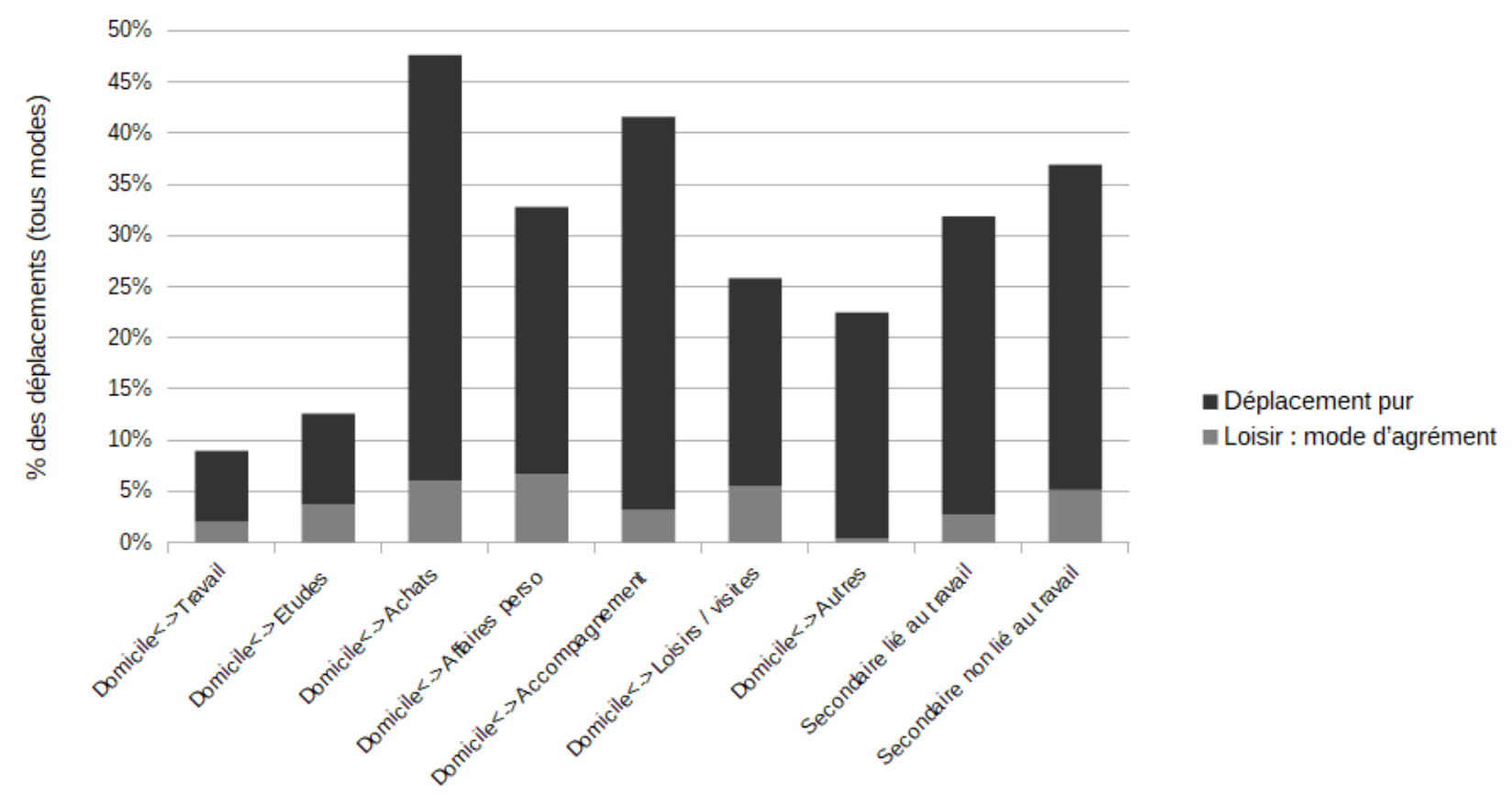

Motifs à l'origine et à la destination des déplacements

Champ : déplacements réalisés par individus majeurs résidant en Ïle-de-France. Source : EGT 2010-STIF-OMNIL-DRIEA. Réalisation : Julie Chrétien

Ainsi, lorsque la pression temporelle est plus forte et que le temps représente un coût plus élevé, on observe une baisse de la marche en tant que mode d'agrément. La pratique de la marche utilitaire, elle, demeure régie par des logiques liées aux distances à parcourir : elle est fortement pratiquée lorsque les destinations sont proches et que la marche est le mode le plus optimal temporellement pour se déplacer-accompagnement des enfants à l'école, pause-déjeuner au milieu d'une journée de travail -, mais est abandonnée lorsque les destinations sont lointaines et que les modes motorisés permettent des gains en temps non négligeables.

Ces résultats sont confirmés si l'on observe la contrainte posée par l'emploi du temps de l'individu dans son ensemble (Figure 3). Les personnes qui font le moins de marche-loisir sont les étudiants et les actifs occupés ayant travaillé le jour de l'enquête, tandis que les personnes qui ont moins de contraintes ce jour-là - à savoir les retraités, chômeurs, actifs en congés et les personnes sans profession - pratiquent bien plus fréquemment la marche en tant qu'activité à part entière. Ainsi, les pratiques de marche-loisir représentent $31 \%$ des déplacements piétons des retraités, contre seulement $16 \%$ de ceux des actifs ayant travaillé le jour de l'enquête. Par ailleurs, la part modale de la marche dans son ensemble décroît lorsque les contraintes augmentent : à titre d'exemple, $38 \%$ des déplacements des actifs en congé et $47 \%$ des déplacements des chômeurs sont réalisés à pied, contre $28 \%$ de ceux des étudiants et des actifs ayant travaillé le jour de l'enquête. C'est donc une hausse en valeur relative et absolue des pratiques de marche-loisir à laquelle on assiste lorsque les gens disposent de temps.

Si l'on distingue les individus motorisés des captifs de la marche, la prévalence de la marche-loisir est stable chez les individus ayant un emploi du temps moins contraint, mais varie légèrement parmi les étudiants et les actifs ayant travaillé. Chez les actifs ayant travaillé (respectivement les étudiants), au sein des déplacements piétons

\footnotetext{
${ }^{5}$ À l'exception de l'interversion des domicile-études et domicile-accompagnement, l'ordre demeure inchangé si l'on prend un seuil de 10 minutes.

${ }^{6}$ Ce qui s'explique par les longues distances de ces trajets, longues distances sur lesquelles la marche n'est pas optimale.
} 
(hors promenade) la marche est pratiquée en tant que mode d'agrément dans $10 \%$ (respectivement $14 \%$ ) des cas parmi les motorisés, contre $16 \%$ (respectivement $21 \%$ ) chez les captifs. Les déplacements identifiés comme relevant d'une pratique d'agrément sont donc surestimés chez les personnes ayant un emploi du temps plus contraint, une partie des longs trajets piétons de cette population relevant d'un effort supplémentaire en temps pour pallier l'absence d'alternative modale.

Figure 3 : Type de pratique piétonne en fonction du statut d'occupation des individus réalisant les déplacements

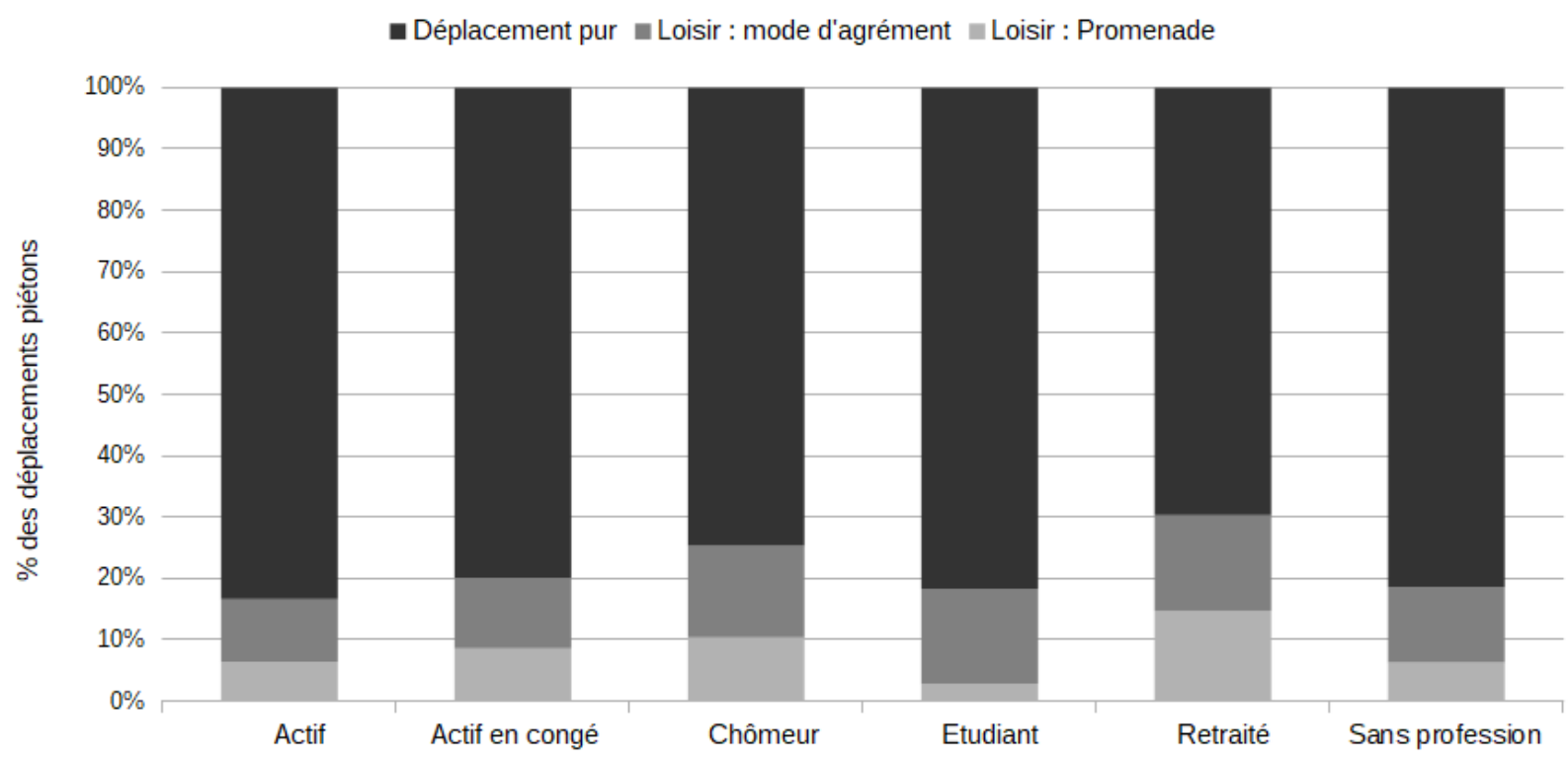

Statut d'occupation des individus

Champ : déplacements réalisés par individus majeurs résidant en lle-de-France. Source : EGT 2010-STIF-OMNIL-DRIEA. Réalisation : Julie Chrétien

La proportion des pratiques de loisirs au sein des déplacements piétons augmente avec le niveau de contrainte temporelle, qu'il s'agisse de promenades ou de l'usage de la marche en tant que mode d'agrément. Lorsque les contraintes temporelles du quotidien sont moins fortes, d'une part les individus ont plus de temps à consacrer aux promenades ${ }^{7}$ et d'autre part le temps a une valeur moins forte ce qui signifie que l'utilité positive associée à la marche est moins fortement contrebalancée par le « coût » que peut représenter le déplacement.

Pour autant, la marche utilitaire demeure la logique largement majoritaire même au sein des populations ayant - théoriquement - du temps à disposition. Si l'on exclut les situations de promenade, sa pratique représente $82 \%$ des déplacements piétons des retraités, qui sont pourtant le groupe ayant la plus faible prévalence de cette pratique. De plus la dispersion des durées des déplacements piétons (hors promenade) révèle que si les actifs ayant travaillé réalisent les déplacements piétons les plus courts en temps et les retraités les plus longs, les écarts entre ces deux populations sont relativement faibles : moins de cinq minutes au niveau de la médiane et du troisième quartile, tandis que, quel que soit le statut d'occupation, le premier quartile est identique. Les personnes qui disposent de temps utilisent donc globalement la marche pour réaliser des déplacements similaires en temps à ceux réalisés par les personnes pressées. Dans la plupart des cas, elles n'entreprennent pas de réaliser à pied des trajets que d'autres populations à l'emploi du temps plus contraint auraient réalisés par un mode motorisé.

\section{DISCUSSION ET CONCLUSION}

Nos travaux ont permis de mieux cerner les poids respectifs des usages utilitaires et de loisir de la marche, l'impact des circonstances spatiales et temporelles des déplacements sur ces pratiques et par extension de renseigner l'importance relative du coût du temps et de la valeur associée à l'expérience du déplacement dans les trajets piétons quotidiens. En semaine, les promenades concernent $9 \%$ des déplacements piétons des adultes d'île-de-France et pour $12 \%$ des déplacements à pied la marche a été choisie par agrément plus que pour son efficacité. Les conditions temporelles, en diminuant ou augmentant le « coût » du temps, peuvent augmenter ou diminuer la prévalence des

\footnotetext{
${ }^{7}$ Incidemment, il a été montré que les promenades faisaient partie des loisirs qui étaient sacrifiés lorsque le sentiment de
} manque de temps augmentait (Degenne et Lebeaux 2003). 
pratiques de marche-loisir : l'utilité intrinsèque au déplacement justifie alors des temps de trajets plus longs, voire justifie le trajet en lui-même. Cependant comme la marche-loisir est minoritaire, cela a peu d'impact sur la pratique de la marche dans son ensemble. En revanche, le lieu du déplacement, par sa densité, influence la prévalence de la marche utilitaire en rendant possible ou non de se rendre à destination à pied en un temps restreint.

Ainsi, la logique très largement majoritaire de la marche en semaine est une logique utilitaire, qui concerne $79 \%$ des déplacements piétons, alors même que cette pratique est occultée dans les représentations sociales. Le recours à des outils et une méthode permettant d'objectiver simultanément les deux facettes de la marche permet ainsi de faire émerger un aspect souvent ignoré : au quotidien, la valeur positive accordée au trajet en lui-même est insuffisante pour compenser l'importance accordée par les individus à la minimisation de leur temps de transport. La marche est majoritairement empruntée lorsqu'elle est le moyen le plus rapide d'accéder à un service ou une activité, et est abandonnée lorsque son utilisation entraînerait un surcoût en temps par rapport à d'autres modes. On comprend là pourquoi en dépit du goût si souvent professé pour ce mode de transport (Kaufmann 2000 ; Mokhtarian et Salomon 2001 ; Flamm 2004), la pratique de la marche chute fortement dès lors que les villes sont construites avec la voiture pour référence : les pratiques de loisirs sont trop marginales pour compenser l'érosion de la marche lorsque celle-ci perd en rapidité.

Bien qu'un élargissement aux populations de visiteurs, et notamment de touristes, eut permis d'aller plus loin, nos résultats invitent à repenser la place prépondérante accordée aux éléments qui favorisent la marche-loisir dans les politiques d'aménagement. Si l'objectif est de favoriser les pratiques piétonnes indépendamment de la logique qui y préside, il est plus efficace de réduire les distances pour accéder aux services et autres aménités quotidiennes que d'améliorer la qualité esthétique ou sécuritaire des environnements piétons ${ }^{8}$. Plus largement, même en se plaçant dans une logique d'ingénierie ayant pour objectif de permettre aux individus de minimiser leur temps de transport, la marche représente un outil essentiel de la mobilité quotidienne.

\footnotetext{
${ }^{8}$ Ces aménagements sont cependant souvent nécessaires pour que les populations les plus vulnérables aient accès à l'espace piéton. En outre, améliorer l'environnement dans lequel se déroulent des trajets utilitaires peut être un objectif louable en soi.
} 


\section{BIBLIOGRAPHIE}

ANABLE, Jillian, et Birgitta GATERSLEBEN. 2005. «All work and no play? The role of instrumental and affective factors in work and leisure journeys by different travel modes ».

Transportation Research Part A: Policy and Practice 39 (2-3) : p. 163-81.

AugOYARD, Jean-François. 1979. Pas à pas : essai sur le cheminement quotidien en milieu urbain. Collection Espacements. Paris : Seuil.

BACQUE, Marie-Hélène, et Sylvie FOL. 2007. «L'inégalité face à la mobilité : du constat à l'injonction ». Revue suisse de sociologie 33 (1) : p. 89-104.

Bostock, Lisa. 2001. "Pathways of Disadvantage? Walking as a Mode of Transport among LowIncome Mothers ». Health \& Social Care in the Community 9 (1) : p. 11-18.

CHRETIEN, Julie. 2015. « La marche dans les schémas quotidiens de mobilité ». Les Cahiers Scientifiques du Transport, $\mathrm{n}^{\mathrm{0}} 67$ : p. 33-54.

COMMENGES, Hadrien. 2013. «L'invention de la mobilité quotidienne : aspects performatifs des instruments de la socio-économie des transports ». Paris 7.

Crozet, Yves. 2005. « Le temps et les transports de voyageurs ». CEMT-ECMT.

Degenne, Alain, et Marie-Odile Lebeaux. 2003. « Le temps des loisirs, le cycle de vie et ses contraintes ». In Regards croisés sur les pratiques culturelles, édité par Olivier Donnat, p. 85-105. Questions de culture. Paris : Documentation française.

FlAMM, Michael. 2004. «La mobilité quotidienne dans la perspective de la conduite de vie ». In Mobilités, fluidités... libertés ?, par Bertrand Montulet et Vincent Kaufmann, p. 71-94. Bruxelles : Publications des Facultes universitaires Saint-Louis.

GENRE-GRANDPIERRE, Cyrille. 2007. « L'accessibilité aux commerces dans l'aire de vie avignonnaise ». Espace populations sociétés, $\mathrm{n}^{\circ}$ 2007/2-3 : 421-32.

GenRe-GrandPIERRE, Cyrille, et Jean-Christophe Foltete. 2003. « Morphologie urbaine et mobilité en marche à pied ». Cybergeo : European Journal of Geography, octobre.

Handy, Susan, Xinyu CaO, et Patricia L. Mokhtarian. 2005. « Correlation or Causality between the Built Environment and Travel Behavior? Evidence from Northern California ». Transportation Research Part D: Transport and Environment 10 (6) : p. 427-44.

Hess, Paul, Anne Moudon, Mary SnYder, et Kiril Stanilov. 1999. " Site Design and Pedestrian Travel ». Transportation Research Record: Journal of the Transportation Research Board 1674 (janvier) : p. 9-19.

KAUFMANN, Vincent. 2000. Mobilité quotidienne et dynamiques urbaines : la question du report modal. Lausanne : Presses Polytechniques et Universitaires Romandes.

LAVADINHO, Sonia, et Yves WINKIN. 2012. Vers une marche plaisir en ville : boîte à outils pour augmenter le bonheur de marcher. Certu.

LEFAUCONNIER, Amélie, et Eric Gantelet. 2005. « Le temps de recherche d'une place de stationnement ». Prédit. ADEME SARECO.

LEJOYEUX, François, et Dominique LAOUSSE. 2010. «La marche à pied, un transport public individuel $\gg$. Les rapports prospectifs 165. RATP.

Lo, Ria Hutabarat. 2009. «Walkability: what is it? » Journal of Urbanism: International Research on Placemaking and Urban Sustainability 2 (2) : p. 145-66.

MokHTARIAN, Patricia L., et Ilan SAlOMON. 2001. « How derived is the demand for travel? Some conceptual and measurement considerations ». Transportation Research Part A: Policy and Practice 35 (8) : p. 695-719. 
MonNET, Jérôme. 2015. «La marche à pied entre loisir et déplacement ». La Géographie - Acta Geographica, $\mathrm{n}^{\mathrm{o}} 1557$ (juin) : p. 12-15.

MotTe-BAumVoL, Benjamin. 2007. «Les populations périurbaines face à l'automobile en grande couronne francilienne ». Norois. Environnement, aménagement, société, $\mathrm{n}^{\mathrm{0}} 205$ (décembre) : p. 53-66.

. 2010. « La dépendance automobile pour l'accès des ménages aux services : Le cas de la grande couronne francilienne ». Revue d'Économie Régionale \& Urbaine décembre (5) : p. 897-919.

PAPON, Francis, et Régis De SOLERE. 2010. «Les modes actifs : marche et vélo de retour en ville ». La revue, Commissariat général au développement durable-Service de l'observation et des statistiques.

SiMON, Gwendal. 2011. «Entre marche et métro, les mouvements intra-urbains des touristes sous le prisme de 1" "adhérence" à Paris et en Île-de-France ». Recherche Transports Sécurité 28 (1) : p. 25-32.

THOMAS, Rachel. 2004. «Quand le pas fait corps et sens avec l'espace. Aspects sensibles et expressifs de la marche en ville ». Cybergeo : European Journal of Geography, mars. 